DOI: $10.15593 /$ perm.mech/2014.3.09

УДК 539.3

\title{
М.А. Ташкинов
}

Пермский национальный исследовательский политехнический университет, Пермь, Россия

\section{СТОХАСТИЧЕСКОЕ МОДЕЛИРОВАНИЕ ПРОЦЕССОВ ДЕФОРМИРОВАНИЯ УПРУГОПЛАСТИЧЕСКИХ КОМПОЗИТОВ СО СЛУЧАЙНЫМ РАСПОЛОЖЕНИЕМ ВКЛЮЧЕНИЙ С ИСПОЛЬЗОВАНИЕМ МОМЕНТНЫХ ФУНКЦИЙ ВЫСОКИХ ПОРЯДКОВ}

\footnotetext{
Целью данной работы является разработка методики вычисления статистических характеристик полей напряжений и деформаций в компонентах структурно-неоднородных сред с учетом микроструктурных параметров.

Многоуровневая иерархия структурно-неоднородных (композиционных) материалов исследуется с помощью понятия представительного объема, когда параметры более высокого масштаба оцениваются или вычисляются на более низком масштабе. Поля напряжений, деформаций и перемещений представлены в виде статистически однородных кусочно-постоянных функций координат.

Аналитические выражения для статистических характеристик структурных полей, такие как средние значения и дисперсии, формируются из решения стохастических краевых задач и содержат структурные многоточечные моментные функции. При этом порядок используемых функций определяется приближением решения стохастической краевой задачи. С помощью метода функций Грина, краевая задача сводится к интегродифференциальному стохастическому уравнению во флуктуациях перемещений. Получено второе приближение решения краевой задачи в упругопластическом случае. Для установления связи между деформациями в компонентах и макроскопическими деформациями организована итерационная процедура.

Получены новые аналитические выражения для статистических характеристик полей напряжений и деформаций в компонентах упругопластических композиционных материалов с использованием второго приближения решения краевой задачи и моментных функций до пятого порядка включительно. Были синтезированы и исследованы модели трехмерной структуры представительного объема материала с эллипсоидальными полидисперсными включениями, для которых построены многоточечные моментные функции до пятого порядка. Получены численные результаты для характеристик процесса деформирования матрицы пористых материалов с полидисперсными эллипсоидальными включениями в случае чистого сдвига для микроструктур с различной объемной долей включений.

Ключевые слова: стохастическая микроструктура, статистические характеристики, композиты, краевая задача, моментные функции, поля напряжений и деформаций, аппроксимация, трехмерные модели, упругопластичность, аналитические выражения.
} 
Perm National Research Polytechnic University, Perm, Russian Federation

\title{
STOCHASTIC MODELLING OF DEFORMATION PROCESS IN ELASTOPLASTIC COMPOSITES WITH RANDOMLY LOCATED INCLUSIONS USING HIGH ORDER CORRELATION FUNCTIONS
}

\begin{abstract}
The aim of this research is to develop the mechanisms of calculation of stress and strain fields statistical characteristics in components of heterogeneous solid media in dependence on microstructural parameters.

Multiscale hierarchy of heterogeneous (composite) materials is investigated using the Representative Volume Element (RVE) concept when parameters of larger scale models are measured or calculated on a smaller scale. Fields of stress, strain and displacements are presented in the form of statistically homogeneous coordinate functions.

Analytical expressions for statistical characteristics of structural fields, such as mean values and dispersions, are formed using solution of stochastic boundary value problems and contain structural multipoint correlation functions. The order of the required correlation functions is determined by the solution of stochastic boundary value problem. The boundary value problem is being reduced to integral-differential equation in fluctuations of displacements. The second approximation of the problem solution is obtained. To determine relation between deformation in components and macroscopic deformation, the iteration procedure was organized.

New analytical expressions and numerical results for statistical characteristics of stress and strain fields in components of elastoplastic composite materials are derived with the second approximation of the boundary value problem and correlation functions up to fifth order. 3D models of representative volume of material microstructure with polydisperse ellipsoidal inclusions are synthesized; the multipoint correlation functions up to fifth order have been obtained for them. Numerical results are obtained for porous composites with polydisperse ellipsoidal inclusions in simple shear state of strain for microstructures with various inclusions volume fraction.

Keywords: stochastic microstructure, statistical characteristics, composites, boundary value problem, correlation functions, stress and strain fields, approximation, three-dimensional models, elastoplasticity, analytical expressions.
\end{abstract}

\section{Введение}

Структурно-неоднородные композиционные материалы все шире применяются в современной технике и индустрии, проблема определения их свойств является крайне актуальной. Одно из главных преимуществ таких материалов заключается в возможности создания наиболее подходящего, уникального по физико-механическим характеристикам материала для каждого конкретного приложения получения путем вариации физических и геометрических параметров его составных компонентов. На стадии разработки новых материалов с целью предсказания их поведения при разрушении необходимо вычислить 
параметры полей напряжений и деформаций для каждого из компонентов материала.

Затраты и усилия, необходимые для экспериментального изучения таких материалов, могут быть существенно сэкономлены при помощи развития методов многомасштабного моделирования, когда эффективные (гомогенизированные) свойства материала определяют при помощи моделирования его структуры на меньших масштабах. Подобные подходы широко представлены для линейных, периодических и детерминистских моделей $[1,2]$. Важным является создание нелинейных микроуровневых моделей композиционных материалов, способных учесть особенности случайной непериодической микроструктуры, а также их последующая интеграция в макромасштабные модели.

Многоуровневая иерархия структурно-неоднородных материалов, как правило, исследуется с помощью понятия представительного объема [1, 3]. В структурно-неоднородных материалах процессы деформации и разрушения зависят от особенностей локальных полей напряжений, при расчете которых важны флуктуации и отклонения от осредненных значений, вызванные неоднородностями на микромасштабе. В этом случае для исследования поведения компонентов конкретного неоднородного материала одним из решений могло бы быть изучение полей напряжений для ансамбля реализаций его микроструктуры $[1,4]$. Другой, более эффективный подход состоит в оценке статистических моментов полей напряжений.

Для определения статистических характеристик в непериодических случайным образом армированных твердых телах используются стохастические подходы, основанные на теории случайных функций [1, 5-8]. Такие подходы предполагают, что на микроструктурном уровне поля напряжений, деформаций и перемещений могут быть представлены в виде статистически однородных кусочно-постоянных функций координат. Эти функции зависят от радиуса-вектора и могут быть разложены на сумму средней составляющей и флуктуации:

$$
\begin{aligned}
& \sigma_{i j}(\vec{r})=\left\langle\sigma_{i j}(\vec{r})\right\rangle+\sigma_{i j}^{\prime}(\vec{r}), \\
& \varepsilon_{i j}(\vec{r})=\left\langle\varepsilon_{i j}(\vec{r})\right\rangle+\varepsilon_{i j}^{\prime}(\vec{r}), \\
& u_{m}(\vec{r})=\left\langle u_{m}(\vec{r})\right\rangle+u_{m}^{\prime}(\vec{r}),
\end{aligned}
$$


где скобки 〈> означают операцию осреднения; (1) - поле напряжений; (2) - поле деформаций, (3) - поле перемещений.

Представленные таким образом поля учитывают случайность взаимного расположения элементов в структуре, а также статистический разброс свойств компонентов.

В качестве характеристик процессов деформирования в компонентах композита могут быть введены многоточечные статистические моменты стохастических полей напряжений и деформаций. Так, на основе моментов первого порядка (иными словами, средних величин) возможно вычисление эффективных упругих характеристик $[1,7,9$, 10]. Моменты более высоких порядков используются для исследования распределения напряжений и деформаций в компонентах [7, 8, 10-14]. В данной работе подобный метод будет проиллюстрирован на примере получения первых двух моментов микромасштабных полей напряжений и деформаций для композитов в упругопластическом случае. Искомые моменты полей напряжений и деформаций определяются из решения краевых задач, при этом статистическая информация о геометрии неоднородной структуры содержится в многоточечных моментных функциях, которые могут быть построены для образцов или моделей материалов $[1,6,8,15]$.

\section{1. Структурные моментные функции и моменты полей напряжений и деформаций}

Сложность структуры композитов на микроуровне является одной из ключевых преград в применении многоуровневых моделей. Такие параметры, как объемная доля включений, ориентация, форма, размеры и распределение включений значительно влияют на эффективные характеристики композиционных материалов [16-19]. Для точного описания внутренней стохастической структуры композитов используются моментные функции высокого порядка, образованные с помощью индикаторной функции $\lambda(\vec{r})[1,7,8,10,20]$. Значения этой функции определяются положением радиуса-вектора $\vec{r}$ внутри представительного объема материала. Так, для двухфазных материалов $\lambda(\vec{r})=0$ - если точка, определяемая радиусом-вектором, находится в матрице, и $\lambda(\vec{r})=1$ - если она находится во включении. Индикаторная функция также может быть разложена в виде средних и флуктуаций, по аналогии с (1)-(3): 


$$
\lambda(\vec{r})=\langle\lambda(\vec{r})\rangle+\lambda^{\prime}(\vec{r}) .
$$

Выражение для $n$-точечной структурной моментной функции $n$-го порядка имеет следующий вид:

$$
\begin{gathered}
K_{\lambda}^{(n)}\left(\vec{r}, \vec{r}_{1}, \ldots, \vec{r}_{n}\right)=\left\langle\lambda^{\prime}(\vec{r}) \lambda^{\prime}\left(\vec{r}_{1}\right) \ldots \lambda^{\prime}\left(\vec{r}_{n}\right)\right\rangle= \\
=\left\langle(\lambda(\vec{r})-\langle\lambda(\vec{r})\rangle)\left(\lambda\left(\vec{r}_{1}\right)-\left\langle\lambda\left(\vec{r}_{1}\right)\right\rangle\right) \ldots\left(\lambda\left(\vec{r}_{n}\right)-\left\langle\lambda\left(\vec{r}_{n}\right)\right\rangle\right)\right\rangle= \\
=\left\langle(\lambda(\vec{r})-p)\left(\lambda\left(\vec{r}_{1}\right)-p\right) \ldots\left(\lambda\left(\vec{r}_{n}\right)-p\right)\right\rangle,
\end{gathered}
$$

где $p$ - объемная доля включений.

Моментные функции зависят только от расстояния между рассматриваемыми точками $\Delta \vec{r}=\left|\vec{r}-\vec{r}_{n}\right|$, поскольку функция $\lambda(\vec{r})$ является статистически однородной и изотропной. Для единообразного представления моментных функций, полученных для различных микроструктур, удобно использовать нормированные значения:

$$
f_{\lambda}^{n}\left(\vec{r}, \vec{r}_{1}, \vec{r}_{2}, \ldots\right)=\frac{K_{\lambda}^{n}\left(\vec{r}, \vec{r}_{1}, \vec{r}_{2}, \ldots\right)}{D_{\lambda}^{(n)}}
$$

где $D_{\lambda}^{(n)}=(1-p)^{(n)} p+(-p)^{(n)}(1-p)$ - центральный момент $n$-го порядка. Шаг $\Delta \vec{r}$ может быть нормирован с помощью осредненного минимального расстояния между включениями $h_{\text {avg }}$.

Для двухкомпонентных композиционных материалов формулы для моментов первого (средних значений) $\left\langle\varepsilon_{i j}\right\rangle$ и второго порядка (дисперсий) $\left\langle\varepsilon_{i j}^{\prime}(\vec{r}) \varepsilon_{\alpha \beta}^{\prime}(\vec{r})\right\rangle$ в матрице $M$ и включениях $I$ в общем виде выражаются через флуктуации стохастических полей (1)-(3) [7]:

$$
\begin{gathered}
\left\langle\varepsilon_{i j}\right\rangle_{I}=e_{i j}+\frac{1}{p}\left\langle\lambda^{\prime}(\vec{r}) \varepsilon_{i j}^{\prime}(\vec{r})\right\rangle, \\
\left\langle\varepsilon_{i j}\right\rangle_{M}=e_{i j}-\frac{1}{1-p}\left\langle\lambda^{\prime}(\vec{r}) \varepsilon_{i j}^{\prime}(\vec{r})\right\rangle, \\
\left\langle\varepsilon_{i j}^{\prime}(\vec{r}) \varepsilon_{\alpha \beta}^{\prime}(\vec{r})\right\rangle_{I}=\left\langle\varepsilon_{i j}^{\prime}(\vec{r}) \varepsilon_{\alpha \beta}^{\prime}(\vec{r})\right\rangle+e_{i j} e_{\alpha \beta}-\left\langle\varepsilon_{i j}\right\rangle_{I}\left\langle\varepsilon_{\alpha \beta}\right\rangle_{I}+ \\
+\frac{1}{p}\left(\left\langle\lambda^{\prime}(\vec{r}) \varepsilon_{i j}^{\prime}(\vec{r}) \varepsilon_{\alpha \beta}^{\prime}(\vec{r})\right\rangle+e_{i j}\left\langle\lambda^{\prime}(\vec{r}) \varepsilon_{\alpha \beta}^{\prime}(\vec{r})\right\rangle+e_{\alpha \beta}\left\langle\lambda^{\prime}(\vec{r}) \varepsilon_{i j}^{\prime}(\vec{r})\right\rangle\right),
\end{gathered}
$$




$$
\begin{gathered}
\left\langle\varepsilon_{i j}^{\prime}(\vec{r}) \varepsilon_{\alpha \beta}^{\prime}(\vec{r})\right\rangle_{M}=\left\langle\varepsilon_{i j}^{\prime}(\vec{r}) \varepsilon_{\alpha \beta}^{\prime}(\vec{r})\right\rangle+e_{i j} e_{\alpha \beta}-\left\langle\varepsilon_{i j}\right\rangle_{M}\left\langle\varepsilon_{\alpha \beta}\right\rangle_{M}- \\
-\frac{1}{1-p}\left(\left\langle\lambda^{\prime}(\vec{r}) \varepsilon_{i j}^{\prime}(\vec{r}) \varepsilon_{\alpha \beta}^{\prime}(\vec{r})\right\rangle+e_{i j}\left\langle\lambda^{\prime}(\vec{r}) \varepsilon_{\alpha \beta}^{\prime}(\vec{r})\right\rangle+e_{\alpha \beta}\left\langle\lambda^{\prime}(\vec{r}) \varepsilon_{i j}^{\prime}(\vec{r})\right\rangle\right), \\
\left\langle\sigma_{i j}\right\rangle_{I}=\left\langle\sigma_{i j}\right\rangle+\frac{1}{p}\left\langle\lambda^{\prime}(\vec{r}) \sigma_{i j}^{\prime}(\vec{r})\right\rangle, \\
\left\langle\sigma_{i j}\right\rangle_{M}=\left\langle\sigma_{i j}\right\rangle-\frac{1}{1-p}\left\langle\lambda^{\prime}(\vec{r}) \sigma_{i j}^{\prime}(\vec{r})\right\rangle, \\
\left\langle\sigma_{i j}^{\prime}(\vec{r}) \sigma_{\alpha \beta}^{\prime}(\vec{r})\right\rangle_{I}=\left\langle\sigma_{i j}^{\prime}(\vec{r}) \sigma_{\alpha \beta}^{\prime}(\vec{r})\right\rangle+\left\langle\sigma_{i j}\right\rangle\left\langle\sigma_{\alpha \beta}\right\rangle-\left\langle\sigma_{i j}\right\rangle_{I}\left\langle\sigma_{\alpha \beta}\right\rangle_{I}+ \\
+\frac{1}{p}\left(\left\langle\lambda^{\prime}(\vec{r}) \sigma_{i j}^{\prime}(\vec{r}) \sigma_{\alpha \beta}^{\prime}(\vec{r})\right\rangle+\left\langle\sigma_{i j}\right\rangle\left\langle\lambda^{\prime}(\vec{r}) \sigma_{\alpha \beta}^{\prime}(\vec{r})\right\rangle+\left\langle\sigma_{\alpha \beta}\right\rangle\left\langle\lambda^{\prime}(\vec{r}) \sigma_{i j}^{\prime}(\vec{r})\right\rangle\right) \\
\left\langle\sigma_{i j}^{\prime}(\vec{r}) \sigma_{\alpha \beta}^{\prime}(\vec{r})\right\rangle_{M}=\left\langle\sigma_{i j}^{\prime}(\vec{r}) \sigma_{\alpha \beta}^{\prime}(\vec{r})\right\rangle+\left\langle\sigma_{i j}\right\rangle\left\langle\sigma_{\alpha \beta}\right\rangle-\left\langle\sigma_{i j}\right\rangle_{M}\left\langle\sigma_{\alpha \beta}\right\rangle_{M}- \\
\frac{1}{1-p}\left(\left\langle\lambda^{\prime}(\vec{r}) \sigma_{i j}^{\prime}(\vec{r}) \sigma_{\alpha \beta}^{\prime}(\vec{r})\right\rangle+\left\langle\sigma_{i j}\right\rangle\left\langle\lambda^{\prime}(\vec{r}) \sigma_{\alpha \beta}^{\prime}(\vec{r})\right\rangle+\left\langle\sigma_{\alpha \beta}\right\rangle\left\langle\lambda^{\prime}(\vec{r}) \sigma_{i j}^{\prime}(\vec{r})\right\rangle\right),
\end{gathered}
$$

где $p$ - объемная доля включений; $e_{i j}-$ заданный постоянный симметричный тензор малых деформаций, определяющий граничные условия на поверхности представительного объема:

$$
\left.u_{i}(\vec{r})\right|_{\vec{r} \in \Gamma_{V}}=e_{i j} r_{j} .
$$

Случайные поля флуктуаций напряжений $\sigma_{i j}^{\prime}(\vec{r})$ и деформаций $\varepsilon_{i j}^{\prime}(\vec{r})$ определяются через градиент флуктуаций перемещений $u_{i, j}^{\prime}(\vec{r})$ :

$$
\begin{gathered}
\varepsilon_{i j}^{\prime}(\vec{r})=\frac{1}{2}\left(u_{i, j}^{\prime}(\vec{r})+u_{j, i}^{\prime}(\vec{r})\right), \\
\sigma_{i j}^{\prime}(\vec{r})=\sigma_{i j}(\vec{r})-\left\langle\sigma_{i j}\right\rangle=C_{i j k l}^{\prime}(\vec{r}) e_{k l}-\left\langle C_{i j k l}^{\prime}(\vec{r}) \varepsilon_{k l}^{\prime}(\vec{r})\right\rangle+C_{i j k l}(\vec{r}) \varepsilon_{k l}^{\prime}(\vec{r}),
\end{gathered}
$$

где $C_{i j k l}(\vec{r})=\left\langle C_{i j k l}(\vec{r})\right\rangle+C_{i j k l}^{\prime}(\vec{r})$ - поле структурных модулей упругости.

Градиент флуктуации поля перемещений $u_{i, j}^{\prime}(\vec{r})$ определяется из решения стохастической краевой задачи. 


\section{2. Упругопластическая стохастическая краевая задача и ее решение во втором приближении}

Краевую задачу для представительного объема с граничными условиями, заданными в перемещениях, образуют следующие соотношения:

$$
\begin{gathered}
\sigma_{i j, j}(\vec{r})=0, \\
\varepsilon_{i j}(\vec{r})=\frac{1}{2}\left(u_{i, j}(\vec{r})+u_{j, i}(\vec{r})\right), \\
\sigma_{i j}(\vec{r})=C_{i j k l}(\vec{r}) \varepsilon_{k l}(\vec{r}), \\
\left.u_{i}(\vec{r})\right|_{\vec{r} \in \Gamma_{V}}=e_{i j} r_{j},
\end{gathered}
$$

которые включают в себя (18) - уравнение равновесия; (19) - соотношения Коши; (20) - уравнение состояния; $C_{i j k l}(\vec{r})$ - тензор структурных модулей упругости. Граничные условия (21) на поверхности представительного объема заданы в перемещениях и обеспечивают однородность макроскопических деформаций; $e_{i j}-$ произвольно заданный постоянный симметричный тензор малых деформаций, $\vec{r}$ - радиусвектор с компонентами $\left(x_{1}, x_{2}, x_{3}\right) ; r_{j}$ - координаты точек на поверхности представительного объема $\Gamma_{V}$.

Тип границы раздела между двумя фазами - идеальный контакт:

$$
\left.u_{i}^{(M)}(\vec{r})\right|_{\vec{r} \in \Gamma_{V_{M}}}=\left.u_{i}^{(I)}\right|_{\vec{r} \in \Gamma_{V_{I}}}(\vec{r}),
$$

где $\Gamma_{V_{M}}-$ внутренняя поверхность матрицы; $\Gamma_{V_{I}}-$ внешняя граница включения.

С помощью метода функций Грина краевая задача (18)-(21) сводится к интегродифференциальному стохастическому уравнению во флуктуациях перемещений $[5,8,21]$. Это уравнение может быть записано в следующей рекуррентной форме:

$$
\frac{\partial u_{i}^{\prime(\chi)}(\vec{r})}{\partial x_{j}}=\int_{V_{1}} \frac{\partial G_{i m}\left(\vec{r}, \vec{r}_{1}\right)}{\partial x_{j}}\left[C_{m n k l}^{\prime}\left(\vec{r}_{1}\right) e_{k l}+C_{m n k l}^{\prime}\left(\vec{r}_{1}\right) \frac{\partial u_{k}^{\prime(\chi-1)}\left(\vec{r}_{1}\right)}{\partial x_{l}}\right]{ }_{1 n} d V_{1},
$$


где $G_{i m}\left(\vec{r}, \vec{r}_{1}\right)$ - функция Грина $[1,14,15] ; \chi$ - порядок аппроксимации. В первом приближении флуктуации перемещений в правой части уравнения (8) принимаются равными нулю [5, 11, 21]. Новое, более точное решение получено в данном исследовании с использованием второго приближения решения краевой задачи, когда в правой части выражения (22) используется аналитическое выражение для решения в первом приближении.

В упругопластическом случае поля структурных модулей упругости могут быть представлены в следующем виде [11]:

$$
C_{i j k l}(\vec{r}, \boldsymbol{\varepsilon})=3 K\left(\vec{r}, j_{\varepsilon}^{(1)}, j_{\varepsilon}^{(2)}\right) V_{i j k l}+2 \mu\left(\vec{r}, j_{\varepsilon}^{(1)}, j_{\varepsilon}^{(2)}\right) D_{i j k l},
$$

где $K\left(\vec{r}, j_{\varepsilon}^{(1)}, j_{\varepsilon}^{(2)}\right)=\lambda(\vec{r}) K_{I}\left(j_{\varepsilon}^{(1)}, j_{\varepsilon}^{(2)}\right)+(1-\lambda(\vec{r})) K_{M}\left(j_{\varepsilon}^{(1)}, j_{\varepsilon}^{(2)}\right)-$ нелинейный объемный модуль; $\mu\left(\vec{r}, j_{\varepsilon}^{(1)}, j_{\varepsilon}^{(2)}\right)=\lambda(\vec{r}) \mu_{I}\left(j_{\varepsilon}^{(1)}, j_{\varepsilon}^{(2)}\right)+(1-\lambda(\vec{r})) \mu_{M}\left(j_{\varepsilon}^{(1)}, j_{\varepsilon}^{(2)}\right)-$ модуль сдвига. Оба модуля являются материальными функциями инвариантов $j_{\varepsilon}^{(1)}, j_{\varepsilon}^{(2)}$ тензора деформаций. $V_{i j k l}$ и $D_{i j k l}-$ шаровая и девиаторная части единичного тензора $I_{i j k l}=V_{i j k l}+D_{i j k l}$.

Инварианты тензоров напряжений и деформаций являются функциями девиаторных частей тензоров:

$$
j_{\sigma}^{(2)}=\sqrt{\widetilde{\sigma}_{i j} \breve{\sigma}_{i j}}, j_{\varepsilon}^{(2)}=\sqrt{\widetilde{\varepsilon}_{i j} \breve{\varepsilon}_{i j}}, j_{\sigma}^{(1)}=\sigma_{i j} \delta_{i j}, j_{\varepsilon}^{(1)}=\varepsilon_{i j} \delta_{i j},
$$

где $\delta_{i j}-$ Дельта Кронекера; $\breve{\varepsilon}_{i j}=\varepsilon_{i j}-1 / 3 \varepsilon_{k k} \delta_{i j}, \breve{\sigma}_{i j}=\sigma_{i j}-1 / 3 \sigma_{k k} \delta_{i j}$.

Девиаторные части могут быть разложены на средние и флуктуации. Так, для тензора деформаций $\breve{\varepsilon}_{i j}=<\breve{\varepsilon}_{i j}>+\breve{\varepsilon}_{i j}^{\prime}$. В дальнейшем будут использованы лишь средние значения инвариантов:

$$
\begin{gathered}
\left\langle j_{\varepsilon}^{(2)}\right\rangle=\sqrt{\left\langle\breve{\varepsilon}_{i j} \breve{\varepsilon}_{i j}\right\rangle}=\sqrt{\left\langle\breve{\varepsilon}_{i j}\right\rangle\left\langle\breve{\varepsilon}_{i j}\right\rangle+\left\langle\breve{\varepsilon}_{i j}^{\prime} \breve{\varepsilon}_{i j}^{\prime}\right\rangle}, \\
\left\langle j_{\varepsilon}^{(1)}\right\rangle=\left\langle\varepsilon_{i j}\right\rangle \delta_{i j}=\left\langle\varepsilon_{i i}\right\rangle .
\end{gathered}
$$

Флуктуациями $\left\langle\breve{\varepsilon}_{i j}^{\prime} \breve{\varepsilon}_{i j}^{\prime}\right\rangle$ в уравнении (24) можно пренебр ечь. Тогда средние значения объемного модуля и модуля сдвига для матрицы и включений в общем случае могут быть записаны в следующем виде: 


$$
\begin{aligned}
& \mu_{I, M}\left(\vec{r}, j_{\varepsilon}^{(1)}, j_{\varepsilon}^{(2)}\right)=\mu_{I, M}\left(\left\langle\varepsilon_{i i}\right\rangle_{I, M}, \Lambda_{I, M}\right), \\
& K_{I, M}\left(\vec{r}, j_{\varepsilon}^{(1)}, j_{\varepsilon}^{(2)}\right)=K_{I, M}\left(\left\langle\varepsilon_{i i}\right\rangle_{I, M}, \Lambda_{I, M}\right),
\end{aligned}
$$

где $\Lambda_{I, M}=\sqrt{\left\langle\breve{\varepsilon}_{i j}\right\rangle_{I, M}\left\langle\breve{\varepsilon}_{i j}\right\rangle_{I, M}}$.

Второе приближение решения (22) в упругопластическом случае примет следующий вид:

$$
\begin{gathered}
u_{i, j}^{\prime(2)}(\vec{r})=e_{k l} \int_{V_{1}} G_{i m, j}\left(\vec{r}, \vec{r}_{1}\right)\left(C_{m n k l}^{\prime} \lambda^{\prime}\left(\vec{r}_{1}\right)\right),{ }_{n} d V_{1}+ \\
+e_{o q} \int_{V_{1} V_{11}} G_{i m, j}\left(\vec{r}, \vec{r}_{1}\right)\left[C_{m n k l}^{\prime} \lambda^{\prime}\left(\vec{r}_{1}\right) G_{k f, l}\left(\vec{r}_{1}, \vec{r}_{11}\right)\left(C_{f s o q}^{\prime} \lambda^{\prime}\left(\vec{r}_{11}\right)\right),{ }_{11 s}\right],_{1 n} d V_{11} d V_{1},
\end{gathered}
$$

где $C_{m n k l}^{\prime}(\vec{r})=\left(C_{m n k l}^{I}\left(\left\langle\varepsilon_{k k}\right\rangle_{I}, \Lambda_{I}\right)-C_{m n k l}^{M}\left(\left\langle\varepsilon_{k k}\right\rangle_{M}, \Lambda_{M}\right)\right) \lambda^{\prime}(\vec{r})$.

В случае когда размеры представительного объема много меньше размеров тела, в качестве функции Грина может быть использован тензор Кельвина-Сомильяны [7]:

$$
G_{m k}\left(\vec{r}, \vec{r}_{1}\right)=A \frac{\delta_{m k}}{\left|\vec{r}-\vec{r}_{1}\right|}+B \frac{\left(r_{m}-r_{1 m}\right)\left(r_{k}-r_{1 k}\right)}{\left|\vec{r}-\vec{r}_{1}\right|^{3}} .
$$

Коэффициенты $A$ и $B$ зависят от нелинейных модулей:

$$
\begin{aligned}
& A=\frac{\left\langle\left[3 K\left(\vec{r}, j_{\varepsilon}^{(1)}, j_{\varepsilon}^{(2)}\right)-7 \mu\left(\vec{r}, j_{\varepsilon}^{(1)}, j_{\varepsilon}^{(2)}\right)\right]\right\rangle}{24 \pi\left\langle\mu\left(\vec{r}, j_{\varepsilon}^{(1)}, j_{\varepsilon}^{(2)}\right)\right\rangle\left\langle\left[3 K\left(\vec{r}, j_{\varepsilon}^{(1)}, j_{\varepsilon}^{(2)}\right)-4 \mu\left(\vec{r}, j_{\varepsilon}^{(1)}, j_{\varepsilon}^{(2)}\right)\right]\right\rangle}, \\
& B=\frac{\left\langle\left[3 K\left(\vec{r}, j_{\varepsilon}^{(1)}, j_{\varepsilon}^{(2)}\right)-\mu\left(\vec{r}, j_{\varepsilon}^{(1)}, j_{\varepsilon}^{(2)}\right)\right]\right\rangle}{24 \pi\left\langle\mu\left(\vec{r}, j_{\varepsilon}^{(1)}, j_{\varepsilon}^{(2)}\right)\right\rangle\left\langle\left[3 K\left(\vec{r}, j_{\varepsilon}^{(1)}, j_{\varepsilon}^{(2)}\right)-4 \mu\left(\vec{r}, j_{\varepsilon}^{(1)}, j_{\varepsilon}^{(2)}\right)\right]\right\rangle},
\end{aligned}
$$

где

$$
\begin{gathered}
\left\langle K\left(\vec{r}, j_{\varepsilon}^{(1)}, j_{\varepsilon}^{(2)}\right)\right\rangle=p\left\langle K_{I}\left(j_{\varepsilon}^{(1)}, j_{\varepsilon}^{(2)}\right)\right\rangle+(1-p)\left\langle K_{M}\left(j_{\varepsilon}^{(1)}, j_{\varepsilon}^{(2)}\right)\right\rangle \\
\left\langle\mu\left(\vec{r}, j_{\varepsilon}^{(1)}, j_{\varepsilon}^{(2)}\right)\right\rangle=p\left\langle\mu_{I}\left(j_{\varepsilon}^{(1)}, j_{\varepsilon}^{(2)}\right)\right\rangle+(1-p)\left\langle\mu_{M}\left(j_{\varepsilon}^{(1)}, j_{\varepsilon}^{(2)}\right)\right\rangle .
\end{gathered}
$$

Для определения статистических моментов в упругопластическом случае необходимо установить связь между деформациями в компонентах (микроструктурными деформациями) $\left\langle\varepsilon_{i j}\right\rangle_{I, M}$ и макро- 
скопическими деформациями $e_{i j}$. Уравнения (7) и (8) содержат средние значения микроструктурных деформаций как в левой, так и в правой части, поэтому для определения макроскопических деформаций может быть организована итерационная процедура:

$$
\left\langle\varepsilon_{i j}\right\rangle_{M}^{(n)}=e_{i j}-\frac{1}{1-p}\left\langle\lambda^{\prime}(\vec{r}) \varepsilon_{i j}^{\prime}(\vec{r})\right\rangle^{(n-1)},\left\langle\varepsilon_{i j}\right\rangle_{I}^{(n)}=e_{i j}+\frac{1}{p}\left\langle\lambda^{\prime}(\vec{r}) \varepsilon_{i j}^{\prime}(\vec{r})\right\rangle^{(n-1)},
$$

где

$$
\begin{gathered}
\left\langle\lambda^{\prime}(\vec{r}) \varepsilon_{i j}^{\prime}(\vec{r})\right\rangle=\frac{1}{2}\left(e_{k l} \bar{C}_{m n k l} \int_{V_{1}}\left(G_{i m, j}\left(\vec{r}, \vec{r}_{1}\right)+G_{j m, i}\left(\vec{r}, \vec{r}_{1}\right)\right) \frac{\partial K_{\lambda}^{(2)}\left(\vec{r}, \vec{r}_{1}\right)}{\partial x_{1 n}} d V_{1}+\right. \\
\left.+e_{o q} \bar{C}_{f s o q} \bar{C}_{m n k l} \int_{V_{1} V_{11}}\left(G_{i m, j}\left(\vec{r}, \vec{r}_{1}\right)+G_{j m, i}\left(\vec{r}, \vec{r}_{1}\right)\right) G_{k f, l}\left(\vec{r}_{1}, \vec{r}_{11}\right) \frac{\partial^{2} K_{\lambda}^{(3)}\left(\vec{r}, \vec{r}_{1}, \vec{r}_{11}\right)}{\partial x_{1 n} \partial x_{11 s}} d V_{11} d V_{1}\right), \\
\bar{C}_{m n k l}=\left(C_{m n k l}^{I}\left(\left\langle\varepsilon_{k k}\right\rangle_{I}, \Lambda_{I}\right)-C_{m n k l}^{M}\left(\left\langle\varepsilon_{k k}\right\rangle_{M}, \Lambda_{M}\right)\right) .
\end{gathered}
$$

На первой итерации компоненты тензора $e_{i j}$ задаются произвольно. Процедура заканчивается при условии $\left|\left\langle\varepsilon_{i j}\right\rangle_{I, M}^{(n)}-\left\langle\varepsilon_{i j}\right\rangle_{I, M}^{(n)}\right|<10^{-9}$. Значения $\left\langle\varepsilon_{i j}\right\rangle_{I, M},\left\langle\lambda^{\prime}(\vec{r}) \varepsilon_{i j}^{\prime}(\vec{r})\right\rangle$ и $e_{i j}$, полученные после этой процедуры, используются для дальнейших вычислений средних значений и дисперсий полей напряжений и деформаций в компонентах.

Смешанные моменты $\left\langle\varepsilon_{i j}^{\prime}(\vec{r}) \varepsilon_{\alpha \beta}^{\prime}(\vec{r})\right\rangle,\left\langle\lambda^{\prime}(\vec{r}) \varepsilon_{i j}^{\prime}(\vec{r}) \varepsilon_{\alpha \beta}^{\prime}(\vec{r})\right\rangle$ выражаются через второе приближение решения (28) следующим образом:

$$
\begin{gathered}
\left\langle\varepsilon_{i j}^{\prime}(\vec{r}) \varepsilon_{\alpha \beta}^{\prime}(\vec{r})\right\rangle=\frac{1}{4} \bar{C}_{m n k l} \bar{C}_{m \phi h}\left(e_{k l} e_{\phi h} D_{\lambda}^{(2)} \int_{V_{1} V_{2}}\left(G_{i m, j}\left(\vec{r}, \vec{r}_{1}\right)+G_{j m, i}\left(\vec{r}, \vec{r}_{1}\right)\right) \times\right. \\
\times\left(G_{\alpha \gamma, \beta}\left(\vec{r}, \vec{r}_{2}\right)+G_{\beta \gamma, \alpha}\left(\vec{r}, \vec{r}_{2}\right)\right) \frac{\partial^{2} f_{\lambda}^{(2)}\left(\vec{r}_{1}, \vec{r}_{2}\right)}{\partial x_{1 n} \partial x_{2 \eta}} d V_{2} d V_{1}+ \\
+e_{k l} e_{b d} \bar{C}_{v w b d} D_{\lambda}^{(3)} \iint_{V_{1} V_{2} V_{21}}\left(G_{i m, j}\left(\vec{r}, \vec{r}_{1}\right)+G_{j m, i}\left(\vec{r}, \vec{r}_{1}\right)\right)\left(G_{\alpha \gamma, \beta}\left(\vec{r}, \vec{r}_{2}\right)+G_{\beta \gamma, \alpha}\left(\vec{r}, \vec{r}_{2}\right)\right) \times \\
\times G_{\phi v, h}\left(\vec{r}_{2}, \vec{r}_{21}\right) \frac{\partial^{3} f_{\lambda}^{(3)}\left(\vec{r}_{1}, \vec{r}_{2}, \vec{r}_{21}\right)}{\partial x_{1 n} \partial x_{2 \eta} \partial x_{21 w}} d V_{21} d V_{2} d V_{1}+
\end{gathered}
$$




$$
\begin{aligned}
& +e_{\phi h} e_{o q} \bar{C}_{f s o q} D_{\lambda}^{(3)} \int_{V_{1}} \int_{V_{2}} \int_{V_{11}}\left(G_{i m, j}\left(\vec{r}, \vec{r}_{1}\right)+G_{j m, i}\left(\vec{r}, \vec{r}_{1}\right)\right)\left(G_{\alpha \gamma, \beta}\left(\vec{r}, \vec{r}_{2}\right)+G_{\beta \gamma, \alpha}\left(\vec{r}, \vec{r}_{2}\right)\right) \times \\
& \times G_{k f, l}\left(\vec{r}_{1}, \vec{r}_{11}\right) \frac{\partial^{3} f_{\lambda}^{(3)}\left(\vec{r}_{1}, \vec{r}_{2}, \vec{r}_{11}\right)}{\partial x_{1 n} \partial x_{2 \eta} \partial x_{11 s}} d V_{11} d V_{2} d V_{1}+ \\
& +e_{o q} e_{b d} \bar{C}_{f s o q} \bar{C}_{v w b d} D_{\lambda}^{(4)} \iint_{V_{1}} \int_{V_{2}} \int_{V_{11}}\left(G_{V_{21}}\left(G_{i m, j}\left(\vec{r}, \vec{r}_{1}\right)+G_{j m, i}\left(\vec{r}, \vec{r}_{1}\right)\right) \times\right. \\
& \times\left(G_{\alpha \gamma, \beta}\left(\vec{r}, \vec{r}_{2}\right)+G_{\beta \gamma, \alpha}\left(\vec{r}, \vec{r}_{2}\right)\right) G_{k f, l}\left(\vec{r}_{1}, \vec{r}_{11}\right) G_{\phi v, h}\left(\vec{r}_{2}, \vec{r}_{21}\right) \times \\
& \left.\times \frac{\partial^{4} f_{\lambda}^{(4)}\left(\vec{r}_{1}, \vec{r}_{2}, \vec{r}_{11}, \vec{r}_{21}\right)}{\partial x_{1 n} \partial x_{2 \eta} \partial x_{11 s} \partial x_{21 w}} d V_{21} d V_{11} d V_{2} d V_{1}\right) \\
& \left\langle\lambda^{\prime}(\vec{r}) \varepsilon_{i j}^{\prime}(\vec{r}) \varepsilon_{\alpha \beta}^{\prime}(\vec{r})\right\rangle= \\
& =\frac{1}{4} \bar{C}_{m n k l} \bar{C}_{m \phi h}\left(e_{k l} e_{\phi h} D_{\lambda}^{(3)} \int_{V_{1} V_{2}}\left(G_{i m, j}\left(\vec{r}, \vec{r}_{1}\right)+G_{j m, i}\left(\vec{r}, \vec{r}_{1}\right)\right) \times\right. \\
& \times\left(G_{\alpha \gamma, \beta}\left(\vec{r}, \vec{r}_{2}\right)+G_{\beta \gamma, \alpha}\left(\vec{r}, \vec{r}_{2}\right)\right) \frac{\partial^{2} f_{\lambda}^{(3)}\left(\vec{r}, \vec{r}_{1}, \vec{r}_{2}\right)}{\partial x_{1 n} \partial x_{2 \eta}} d V_{2} d V_{1}+ \\
& +e_{k l} e_{b d} \bar{C}_{v w b d} D_{\lambda}^{(4)} \int_{V_{1}} \int_{V_{2}} \int_{V_{21}}\left(G_{i m, j}\left(\vec{r}, \vec{r}_{1}\right)+G_{j m, i}\left(\vec{r}, \vec{r}_{1}\right)\right)\left(G_{\alpha \gamma, \beta}\left(\vec{r}, \vec{r}_{2}\right)+G_{\beta \gamma, \alpha}\left(\vec{r}, \vec{r}_{2}\right)\right) \times \\
& \times G_{\phi v, h}\left(\vec{r}_{2}, \vec{r}_{21}\right) \frac{\partial^{3} f_{\lambda}^{(4)}\left(\vec{r}, \vec{r}_{1}, \vec{r}_{2}, \vec{r}_{21}\right)}{\partial x_{1 n} \partial x_{2 \eta} \partial x_{21 w}} d V_{21} d V_{2} d V_{1}+ \\
& +e_{\phi h} e_{o q} \bar{C}_{f s o q} D_{\lambda}^{(4)} \int_{V_{1}} \int_{V_{2}} \int_{V_{11}}\left(G_{i m, j}\left(\vec{r}, \vec{r}_{1}\right)+G_{j m, i}\left(\vec{r}, \vec{r}_{1}\right)\right)\left(G_{\alpha \gamma, \beta}\left(\vec{r}, \vec{r}_{2}\right)+G_{\beta \gamma, \alpha}\left(\vec{r}, \vec{r}_{2}\right)\right) \times \\
& \times G_{k f, l}\left(\vec{r}_{1}, \vec{r}_{11}\right) \frac{\partial^{3} f_{\lambda}^{(4)}\left(\vec{r}, \vec{r}_{1}, \vec{r}_{2}, \vec{r}_{11}\right)}{\partial x_{1 n} \partial x_{2 \eta} \partial x_{11 s}} d V_{11} d V_{2} d V_{1}+ \\
& +e_{o q} e_{b d} \bar{C}_{f s o q} \bar{C}_{v w b d} D_{\lambda}^{(5)} \iint_{V_{1}} \int_{V_{2}} \int_{V_{11}}\left(G_{21} G_{i m, j}\left(\vec{r}, \vec{r}_{1}\right)+G_{j m, i}\left(\vec{r}, \vec{r}_{1}\right)\right)\left(G_{\alpha \gamma, \beta}\left(\vec{r}, \vec{r}_{2}\right)+G_{\beta \gamma, \alpha}\left(\vec{r}, \vec{r}_{2}\right)\right) \times \\
& \left.\times G_{k f, l}\left(\vec{r}_{1}, \vec{r}_{11}\right) G_{\phi v, h}\left(\vec{r}_{2}, \vec{r}_{21}\right) \frac{\partial^{4} f_{\lambda}^{(5)}\left(\vec{r}, \vec{r}_{1}, \vec{r}_{2}, \vec{r}_{11}, \vec{r}_{21}\right)}{\partial x_{1 n} \partial x_{2 \eta} \partial x_{11 s} \partial x_{21 w}} d V_{21} d V_{11} d V_{2} d V_{1}\right),
\end{aligned}
$$

где $f_{\lambda}^{(2)}\left(\vec{r}, \vec{r}_{1}\right) ; f_{\lambda}^{(3)}\left(\vec{r}, \vec{r}_{1}, \vec{r}_{2}\right) ; f_{\lambda}^{(4)}\left(\vec{r}, \vec{r}_{1}, \vec{r}_{2}, \vec{r}_{11}\right) ; f_{\lambda}^{(5)}\left(\vec{r}, \vec{r}_{1}, \vec{r}_{2}, \vec{r}_{11}, \vec{r}_{21}\right)$ - нормированные структурные моментные функции второго, третьего, четвертого и пятого порядков. 


\section{3. Реализация методики и численные результаты}

В качестве примера реализации предложенной методики были рассмотрены пористые композиты с эллипсоидальными полидисперсными включениями и физически нелинейной изотропной матрицей. В случае пористых композитов достаточно рассмотреть поведение матрицы, поскольку предполагается, что включения содержат вакуум.

Неупругое поведение матрицы в таких композитах рассматривается с позиций теории малых упругопластических деформаций [22]. В рамках теории модуль сдвига является функцией только от второго инварианта тензора микронапряжений. Матрица имеет упругую область, а также область с линейным упрочнением, точка перехода соответствует пределу текучести:

$$
\mu_{M}\left(j_{\varepsilon}^{(2)}\right)= \begin{cases}j_{\varepsilon}^{(2)}<j_{\varepsilon_{\mathrm{T}}}^{(2)}, & G_{M}, \\ j_{\varepsilon}^{(2)}>j_{\varepsilon_{\mathrm{T}}}^{(2)}, & G_{M}\left[\frac{G_{M}^{\prime}}{G_{M}}-\frac{\left(G_{M}^{\prime}-G_{M}\right)}{G_{M}} \frac{j_{\varepsilon_{T}}^{(2)}}{j_{\varepsilon}^{(2)}}\right],\end{cases}
$$

где $G_{M}$ - модуль сдвига матрицы; $G_{M}^{\prime}$ - модуль упрочнения матрицы; $j_{\varepsilon_{T}}^{(2)}-$ инвариант тензора деформаций, соответствующий пределу упругости. Предполагается, что матрица несжимаема, поэтому модуль объемного сжатия постоянен на всем протяжении процесса деформирования.

Для расчетов было выбрано напряженно-деформированное состояние представительного объема, соответствующее чистому сдвигу. Были заданы следующие ненулевые компоненты тензора макродеформаций $e_{i j}$ в граничных условиях (21): $e_{12}=e_{21}=10^{-6}$. Учитывая формулы для девиаторных частей, инварианты тензоров напряжений и деформаций для случая чистого сдвига можно выразить через средние значения полей напряжений и деформаций в матрице:

$$
\Lambda_{M}=\sqrt{2\left\langle\varepsilon_{12}\right\rangle_{M}\left\langle\varepsilon_{12}\right\rangle_{M}}, j_{\sigma}^{(2)}\left(\Lambda_{M}\right)=\sqrt{2\left\langle\sigma_{12}\right\rangle_{M}\left\langle\sigma_{12}\right\rangle_{M}} .
$$

Механические свойства матрицы были заданы в следующем виде:

$$
\begin{gathered}
G_{M}=44,2 \mathrm{GPa}, G_{M}^{\prime}=14,3 \mathrm{Gpa}, \\
K_{M}=132,2 \mathrm{Gpa}, j_{\varepsilon_{T}}^{(2)}=6,37 \times 10^{-4} .
\end{gathered}
$$


Геометрия микроструктуры была получена с помощью трехмерного моделирования. Структуры с полидисперсными непересекающимися эллипсоидальными включениями синтезированы в программном комплексе Wolfram Mathematica. Алгоритм синтеза состоял из следующих шагов:

1. Генерация произвольных координат точки пересечения полуосей эллипсоида в пределах представительного объема (в данном примере - куба со стороной $a=200$ ).

2. Генерация произвольных размеров полуосей эллипсоида в рамках установленного интервала.

3. Выбор ориентации главной полуоси.

4. Проверка пересечения описанных вокруг эллипсоидов сфер.

Для реализации заданной объемной доли включений была применена модель твердого ядра [23]. Такой алгоритм позволяет генерировать разреженные структуры с эллипсоидальными включениями объемной долей до $\approx 22 \%$. Геометрические параметры полученных и исследованных в данной работе микроструктур приведены в табл. 1. Все размеры представлены в некоторых условных величинах, поскольку метод и все уравнения не зависят от реального размера структур.

Таблица 1

Геометрические параметры синтезированных структур

\begin{tabular}{|l|c|c|c|}
\hline \multirow{2}{*}{\multicolumn{1}{|c|}{ Параметры }} & \multicolumn{3}{c|}{ Объемная доля } \\
\cline { 2 - 4 } & 0,15 & 0,18 & 0,20 \\
\hline Количество включений & 48 & 81 & 87 \\
\hline Минимальный размер полуоси эллипсоида & \multicolumn{3}{|c|}{48} \\
\hline Максимальный размер полуоси эллипсоида & 53,63 & 46,55 & 46,52 \\
\hline $\begin{array}{l}\text { Осредненное минимальное расстояние между } \\
\text { включениями }\end{array}$ & & & \\
\hline
\end{tabular}

Визуализация синтезированной структуры с объемной долей включений 20 \% представлена на рис. 1.

Значения моментных функций для исследуемых структур были получены с использованием сеточных методов: фрагмент синтезированной стохастической структуры был разбит сеткой, при этом поле значений индикаторной функции определялось наличием матрицы или одного из включений в узлах сетки [5]. 


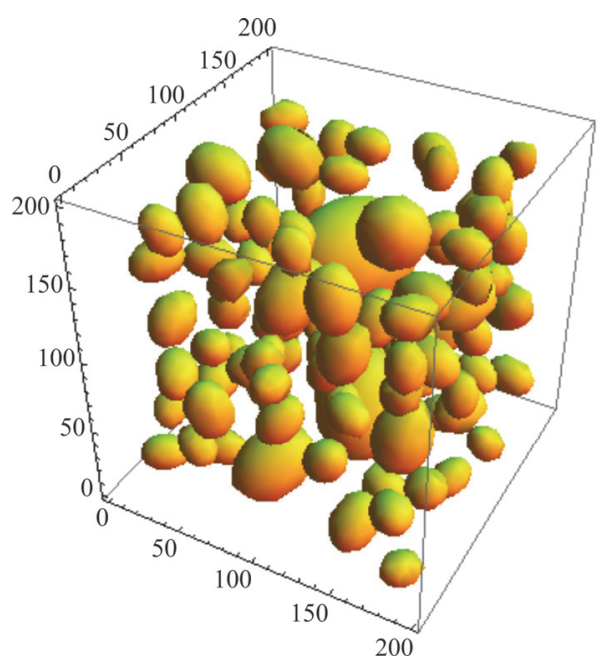

Рис. 1. Модель стохастической структуры с эллипсоидальными включениями объемной доли $20 \%$

Для аппроксимации многоточечных моментных функций порядка выше второго было использовано следующее выражение:

$$
f_{\lambda}^{(n)}\left(\vec{r}, \vec{r}_{1}, \vec{r}_{2}, \ldots, \vec{r}_{n-1}\right)=\exp \left(-c_{1} \frac{\Sigma_{n}}{h_{\text {avg }}}\right)\left[\cos \left(c_{2} \frac{\Sigma_{n}^{(2)}}{h_{\text {avg }}^{2}}\right)\right],
$$

где $\Sigma_{n}=\left|\vec{r}-\vec{r}_{1}\right|+\left|\vec{r}-\vec{r}_{2}\right|+\ldots+\left|\vec{r}-\vec{r}_{n-1}\right| ; \quad \Sigma_{n}^{(2)}=\left|\vec{r}-\vec{r}_{1}\right|^{2}+\left|\vec{r}-\vec{r}_{2}\right|^{2}+\ldots+\left|\vec{r}-\vec{r}_{n-1}\right|^{2} ;$ $c_{i}$ - коэффициенты аппроксимации; $h_{\text {avg }}$ - осредненное по количеству включений расстояние между центром включения до ближайшего включения.

Графики моментных функций второго порядка (корреляционных функций) для некоторых структур могут иметь область отрицательных значений, характеризующих периодичность структуры. Эта особенность может быть достаточно точно аппроксимирована классом выражений с двумя периодическими функциями и тремя коэффициентами $[7,8]$ :

$$
f_{\lambda}^{(2)}\left(\vec{r}, \vec{r}_{1}\right)=\exp \left(c_{1}, \vec{r}, \vec{r}_{1}\right)\left[\cos \left(c_{2}, \vec{r}, \vec{r}_{1}\right)+c_{3} \sin \left(c_{2}, \vec{r}, \vec{r}_{1}\right)\right] .
$$

В данной работе используется следующая зависимость для аппроксимации моментных функций второго порядка: 


$$
f_{\lambda}^{(2)}\left(\vec{r}, \vec{r}_{1}\right)=\exp \left(-c_{1} \frac{\left|\vec{r}-\vec{r}_{1}\right|}{h_{\text {avg }}}\right)\left[\cos \left(c_{2} \frac{\left|\vec{r}-\vec{r}_{1}\right|}{h_{\text {avg }}}\right)+c_{3} \sin \left(c_{2} \frac{\left|\vec{r}-\vec{r}_{1}\right|^{2}}{h_{\text {avg }}^{2}}\right)\right] .
$$

Для вычисления коэффициентов в Wolfram Mathematica была реализована автоматизированная процедура с использованием линейного метода сопряженных градиентов. Коэффициенты аппроксимирующих выражений для моментных функций, построенных для исследуемых структур, представлены в табл. 2.

Таблица 2

Коэффициенты аппроксимирующих выражений для моментных функций

\begin{tabular}{|c|c|c|c|c|c|}
\hline & & $\begin{array}{c}\text { Моментная } \\
\text { функция } \\
\text { второго } \\
\text { порядка } \\
\end{array}$ & $\begin{array}{c}\text { Моментная } \\
\text { функция } \\
\text { третьего } \\
\text { порядка }\end{array}$ & $\begin{array}{c}\text { Моментная } \\
\text { функция } \\
\text { четвертого } \\
\text { порядка } \\
\end{array}$ & $\begin{array}{c}\text { Моментная } \\
\text { функция } \\
\text { пятого } \\
\text { порядка } \\
\end{array}$ \\
\hline Структура с & $c_{1}$ & 110,5733 & 4,7227 & 4,8799 & 4,8496 \\
\hline $\begin{array}{c}\text { объемной долей } \\
p=0,15\end{array}$ & $c_{2}$ & $-185,0099$ & $-5,0204$ & $-3,6068$ & $-3,8547$ \\
\hline & $c_{3}$ & $-26,4138$ & - & - & - \\
\hline Структура с & $c_{1}$ & 108,1408 & 4,9258 & 4,9338 & 4,9331 \\
\hline $\begin{array}{c}\text { объемной долей } \\
p=018\end{array}$ & $c_{2}$ & $-160,0396$ & 5,5577 & 3,7463 & 3,8458 \\
\hline & $c_{3}$ & $-24,0798$ & - & - & - \\
\hline Структура с & $c_{1}$ & 105,1644 & 4,7296 & 4,7433 & 4,7644 \\
\hline объемной долей & $c_{2}$ & $-160,5884$ & $-3,9278$ & $-2,2565$ & $-2,7738$ \\
\hline & $c_{3}$ & $-23,9662$ & - & - & - \\
\hline
\end{tabular}

Интегральные уравнения в выражениях (7)-(14) были вычислены в Wolfram Mathematica с использованием численных методов и параллельных вычислений.

График зависимости средних напряжений в матрице от макродеформаций представительного объема представлен на рис. 2 для всех исследованных структур.

На рис. 3 и 4 представлены зависимости дисперсий напряжений и деформаций в матрице от макроскопических напряжений. 


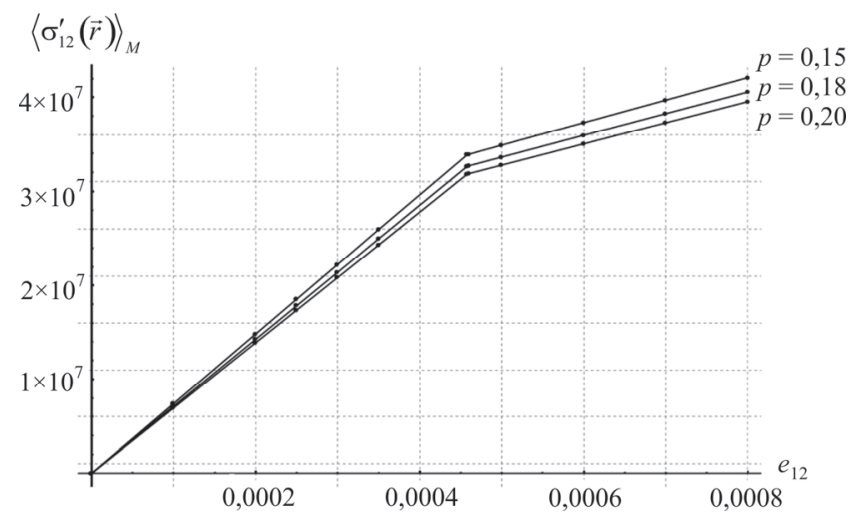

Рис. 2. Зависимость средних напряжений в матрице от макродеформаций представительного объема

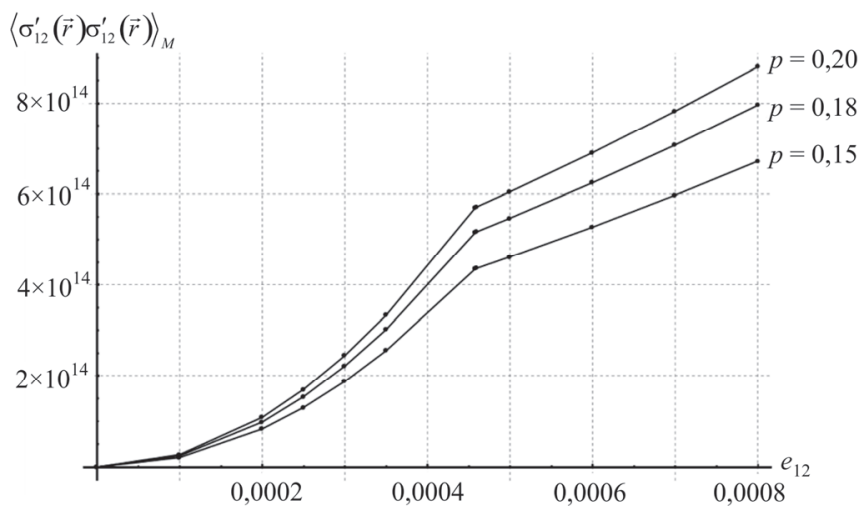

Рис. 3. Зависимость дисперсий напряжений в матрице от макродеформаций представительного объема

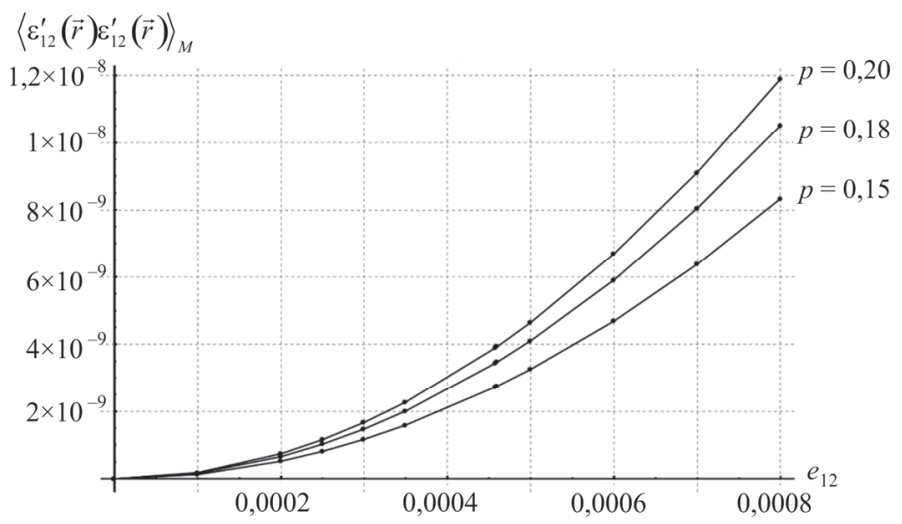

Рис. 4. Зависимость дисперсий деформаций в матрице от макродеформаций представительного объема 
Полученные результаты демонстрируют возможность применения методов стохастической механики для анализа полей напряжений и деформаций в отдельных компонентах структурно-неоднородных материалов. Геометрические особенности неоднородной микроструктуры учтены с помощью структурных моментных функций, следовательно, полученные статистические характеристики позволяют определять характерные особенности материалов определенного класса без необходимости расчета многих реализаций его случайной микроструктуры, что значительно сокращает необходимые вычислительные затраты при решении задач микромеханики.

\section{Заключение}

Впервые аналитические выражения для моментов микроструктурных напряжений и деформаций в компонентах неоднородных твердых тел были получены во втором приближении решения краевой задачи для упругопластического случая. Было показано, что интегральные уравнения для моментов первого и второго порядка зависят от многоточечных структурных моментных функций до пятого порядка.

Рассмотрен упругопластический случай, в котором модули сдвига и сжатия были представлены через первый и второй инварианты тензора деформаций. Связь между микро- и макродеформациями установлена с помощью итерационной процедуры.

В качестве примера реализации методики были рассмотрены пористые композиты, макроскопическое напряженно-деформированное состояние представительного объема было задано в виде чистого сдвига. Матрица материала считалась изотропной и физически нелинейной. Внутренняя геометрия микроструктуры задавалась с помощью синтезированных трехмерных случайных структур с непересекающимися эллипсоидальными включениями. Объемная доля включений в структурах варьировалась от 15 до $20 \%$. Для таких структур были построены моментные функции вплоть до пятого порядка, найдены коэффициенты аппроксимирующих выражений для них.

Получены зависимости статистических характеристик полей напряжений и деформаций в матрице от макродеформаций представительного объема.

Были разработаны новые автоматизированные алгоритмы в Wolfram Mathematica, которые позволяют выполнять полный цикл 
необходимых вычислений для предложенной методологии, от синтеза структур и построения моментных функций для них до расчета статистических характеристик полей напряжений и деформаций в различных фазах композитов. Примененные методы параллельных вычислений значительно сократили время вычислений.

Разработанные методы моделирования микроструктуры и решений краевых задач стохастической механики могут быть использованы для сравнительного анализа влияния различных структурных параметров на статистические характеристики полей напряжений с целью создания материалов с заранее определенным набором свойств и оценки вероятности их разрушения.

Работа выполнена при поддержке Российского фонда фундаментальных исследований, грант № 14-01-96024-р_урал_а.

\section{Библиографический список}

1. Buryachenko V. Micromechanics of heterogeneous materials. New York: Springer, 2007. -686 p.

2. Torquato S. Random heterogenous materials, microstructure and macroscopic properties. - Springer, 2001. $-701 \mathrm{p}$.

3. Determination of the size of the representative volume element for random composites: statistical and numerical approach / T. Kanit, S. Forest, I. Galliet, V. Mounoury, D. Jeulin // International Journal of Solids and Structures. - 2003. - Vol. 40. - P. 3647-3679.

4. Liu K.C., Ghoshal A. Validity of random microstructures simulation in fiber-reinforced composite materials // Composites Part B: Engineering. - 2014. - Vol. 57. - P. 56-70.

5. Tashkinov M.A., Wildemann V.E., Mikhailova N.V. Method of successive approximations in stochastic elastic boundary value problem for structurally heterogenous materials // Computational Materials Science. 2012. - Vol. 52. - P 101-106. DOI: 10.1016/j.commatsci.2011.04.025

6. Хорошун Л.П. Методы случайных функций в задачах о макроскопических свойствах микронеоднородных сред // Прикл. механика. 1978. - Т. 14. - Вып. 2. - С. 3-17.

7. Волков С.Д., Ставров В.П. Статистическая механика композитных материалов. - Минск: Изд-во Белорус. гос. ун-та, 1978. - 208 с. 
8. Вильдеман В.Э., Соколкин Ю.В., Ташкинов А.А. Механика неупругого деформирования и разрушения композиционных материалов. М.: Наука, 1997. - 288 с.

9. Saheli G., Garmestani H., Adams B.L. Microstructure design of a two phase composite using two-point correlation functions // Journal of Computer-Aided Materials Design. - 2004. - Vol. 11. - P. 103-115.

10. Шермергор Т.Д. Теория упругости микронеоднородных сред. М.: Наука, 1976. - 400 с.

11. Михайлова Н.В., Ташкинов А.А. Упругопластическое деформирование дисперсных композитов с разреженной случайной структурой // Механика композиционных материалов и конструкций. - 2010. Т. 16, № 4. - С. 469-482.

12. Паньков А.А. Статистическая механика пьезокомпозитов. Пермь: Изд-во Перм. гос. техн. ун-та, 2009. - 480 с.

13. Соколкин Ю.В., Волкова Т.А. Расчет распределения деформаций и напряжений в зернистых композитах с учетом реальных моментных функций свойств микроструктуры // Механика композиционных материалов и конструкций. - 1998. - Т. 4, № 3. - С. 70-85.

14. Соколкин Ю.В., Ташкинов А.А. Механика деформирования и разрушения структурно-неоднородных тел. - М.: Наука, 1984. - 116 с.

15. Jiao Y., Stillinger F.H., Torquato S. Modeling heterogeneous materials via two-point correlation functions. II. Algorithmic details and applications // Physical Review. -2008. - Vol. 77. - No. 3. - P. 031135. DOI: 10.1103/PhysRevE.77.031135

16. Kaminski M.M. Computational mechanics of composite materials. Springer, 2005. $-433 \mathrm{p}$.

17. Multiscale methods for composites: a review / P. Kanouté, D.P. Boso, J.L. Chaboche, B.A. Schrefler // Arch. Comput. Methods. Eng. - 2009. Vol. 16. - P. 31-75.

18. Ильиных А.В., Радионова М.В., Вильдеман В.Э. Компьютерный синтез и статистический анализ распределения структурных характеристик зернистых композиционных материалов // Механика композиционных материалов и конструкций. -2010 . - Т. 16, № 2. - С. 251265.

19. Rasool A., Böhm H.J. Effects of particle shape on the macroscopic and microscopic linear behaviors of particle reinforced composites // International Journal of Engineering Science. - 2012. - Vol. 58. - P. 21-34. 
20. Hori M., Kubo J. Analysis of probabilistic distribution and range of average stress in each phase of heterogeneous materials // J. Mech. Phys. Solids. - 1998. - Vol. 46. - P. 537-556.

21. Tashkinov M.A., Vildeman V.E., Mikhailova N.V. Method of successive approximations in a stochastic boundary-value problem in the elasticity theory of structurally heterogeneous media // Composites: Mechanics, Computations, Applications. - 2011. - Vol. 2. - No. 1. - P. 21-37.

22. Silberschmidt V.V. Account for random microstructure in multiscale models // Multiscale Modeling and Simulation of Composite Materials and Structures. Eds. Y.W. Kwon, D.H. Allen and R. Talreja. - New York: Springer, 2008. - P. 1-35.

23. Lotwick H.W. Simulations on some spatial hard core models, and the complete packing problem // J. Statist. Comp. Simul. - 1982. - Vol. 15. P. 295-314.

\section{References}

1. Buryachenko V. Micromechanics of heterogeneous materials. New York: Springer, 2007, $686 \mathrm{p}$.

2. Torquato S. Random heterogenous materials, microstructure and macroscopic properties. Springer, 2001, $701 \mathrm{p}$.

3. Kanit T., Forest S., Galliet I., Mounoury V., Jeulin D. Determination of the size of the representative volume element for random composites: statistical and numerical approach. International Journal of Solids and Structure, 2003, vol. 40, pp. 3647-3679.

4. Liu K.C., Ghoshal A. Validity of random microstructures simulation in fiber-reinforced composite materials. Composites Part B: Engineering, 2014, vol. 57, pp. 56-70.

5. Tashkinov M.A., Wildemann V.E., Mikhailova N.V. Method of successive approximations in stochastic elastic boundary value problem for structurally heterogenous materials. Computational Materials Science. 2012, vol. 52, pp. 101-106. DOI: 10.1016/j.commatsci.2011.04.025

6. Khoroshun L.P. Metody sluchaynykh funktsiy $\mathrm{v}$ zadachakh o makroskopicheskikh svoystvakh mikroneodnorodnykh sred [Methods of random functions in the problems of macroscopic properties of microinhomogeneous media]. Prikl. mehanika, 1978, vol. 14, no. 2, pp. 3-17.

7. Volkov S.D., Stavrov V.P. Statisticheskayia mekhanika kompozitnykh materialov [Statistical mechanics of composite materials]. Minsk: Belorusskii gosudarstvennyi universitet, 1978, $208 \mathrm{p}$. 
8. Vil'deman V.E., Sokolkin Yu.V., Tashkinov A.A. Mehanika neuprugogo deformirovaniia i razrusheniia kompozicionnykh materialov [Mechanics of nonlinear deformation and failure of composite materials]. Moscow: Nauka, 1997. 288 p.

9. Saheli G., Garmestani H., Adams B.L. Microstructure design of a two phase composite using two-point correlation functions. Journal of Computer-Aided Materials Design, 2004, vol. 11, pp. 103-115.

10. Shermergor T.D. Teoriia uprugosti mikroneodnorodnykh sred [Elasticity theory of microinhomogeneous media]. Moscow: Nauka, 1976. 400 p.

11. Mikhailova N.V., Tashkinov A.A. Uprugoplasticheskoe deformirovanie dispersnykh kompozitov $\mathrm{s}$ razrezhennoy sluchainoy strukturoy [Elastoplastic deformation of disperse composites with sparse random structure]. Mehanika kompozicionnyh materialov i konstrukciy, 2010, vol. 16, no. 4, pp. 469-482.

12. Pan'kov A.A. Statisticheskaya mehanika piezokompozitov [Statistical mechanics of piezocomposites]. Permskii gosudarstvennyi tekhnicheskii universitet, 2009. $480 \mathrm{p}$.

13. Sokolkin Yu.V., Volkova T.A. Raschet raspredeleniia deformatsii i napriazhenii v zernistykh kompozitakh s uchetom real'nykh momentnykh funktsii svoistv mikrostruktury [Calculation of stress and strain distribution in granular composites with real moment functions of microstructure properties]. Mekhanika kompozitsionnykh materialov i konstruktsii, 1998, vol. 4, no. 3, pp. 70-85.

14. SokolkinYu.V., Tashkinov A.A. Mekhanika deformirovaniia i razrusheniia strukturno-neodnorodnykh tel [Mechanics of deformation and failure of structurally heterogeneous bodies]. Moscow: Nauka, 1984. 116 p.

15. Jiao Y., Stillinger F.H., Torquato S. Modeling heterogeneous materials via two-point correlation functions. II. Algorithmic details and applications. Physical Review, 2008, vol. 77, no. 3, p. 031135. DOI: 10.1103/PhysRevE.77.031135.

16. Kaminski M.M. Computational mechanics of composite materials. Springer, 2005. $433 \mathrm{p}$.

17. Kanouté P., Boso D.P., Chaboche J.L., Schrefler B.A. Multiscale methods for composites: a review. Arch. Comput. Methods. Eng., 2009, vol. 16, pp. 31-75.

18. Il'inykh A.V., Radionova M.V., Vil'deman V.E. Kompyuternyi sintez i statisticheskii analiz raspredeleniia strukturnykh kharakteristik zern- 
istykh kompozitsionnykh materialov [Computer synthesis and statistical analysis of distribution of structural characteristics of granular composite materials]. Mekhanika kompozitsionnykh materialov i konstruktsii, 2010, vol. 16, no. 2, pp. 251-265.

19. Rasool A., Böhm H.J. Effects of particle shape on the macroscopic and microscopic linear behaviors of particle reinforced composites. International Journal of Engineering Science, 2012, vol. 58, pp. 21-34.

20. Hori M., Kubo J. Analysis of probabilistic distribution and range of average stress in each phase of heterogeneous materials. J. Mech. Phys. Solids, 1998, vol. 46, pp. 537-556.

21. Tashkinov M.A., Vildeman V.E., Mikhailova N.V. Method of successive approximations in a stochastic boundary-value problem in the elasticity theory of structurally heterogeneous media. Composites: Mechanics, Computations, Applications, 2011, vol. 2, no. 1, pp. 21-37.

22. Silberschmidt V.V. Account for random microstructure in multiscale models. In: Multiscale Modeling and Simulation of Composite Materials and Structures. Eds. Y.W. Kwon, D.H. Allen and R. Talreja, New York: Springer, 2008, pp. 1-35.

23. Lotwick H.W. Simulations on some spatial hard core models, and the complete packing problem. J. Statist. Comp. Simul., 1982, vol. 15, pp. 295-314.

\section{Об авторах}

Ташкинов Михаил Анатольевич (Пермь, Россия) - кандидат физико-математических наук, старший научный сотрудник Центра экспериментальной механики Пермского национального исследовательского политехнического университета (614990, г. Пермь, Комсомольский пр., 29, e-mail: tma@pstu.ru).

\section{About the authors}

Mikhail A. Tashkinov (Perm, Russian Federation) - PhD in Physical and Mathematical Sciences, Senior Researcher The Center of Experimental Mechanics, Perm National Research Polytechnic University (29, Komsomolsky av., 614990, Perm, Russian Federation, e-mail: tma@pstu.ru).

Получено 04.08.2014 
Просьба ссылаться на эту статью в русскоязычных источниках следующим образом:

Ташкинов М.А. Стохастическое моделирование процессов деформирования упругопластических композитов со случайным расположением включений с использованием моментных функций высоких порядков // Вестник Пермского национального исследовательского политехнического университета. Механика. - 2014. - № 3. C. 163-185. DOI: 10.15593/perm.mech/2014.3.09

Please cite this article in English as:

Tashkinov M.A. Stochastic modeling of deformation process in elastoplastic composites with randomly located inclusions using high order correlation functions. PNRPU Mechanics Bulletin. 2014. No. 3. Pp. 163-185. DOI: 10.15593/perm.mech/2014.3.09 